\title{
DSpace@MIT
}

\author{
MIT Open Access Articles
}

Grain boundary segregation in Al-Mn electrodeposits prepared from ionic liquid

The MIT Faculty has made this article openly available. Please share how this access benefits you. Your story matters.

Citation: Huang, Ting-Yun, Christopher J. Marvel, Patrick R. Cantwell, Martin P. Harmer, and Christopher A. Schuh. "Grain Boundary Segregation in Al-Mn Electrodeposits Prepared from Ionic Liquid.” Journal of Materials Science 51, no. 1 (August 8, 2015): 438-448.

As Published: http://dx.doi.org/10.1007/s10853-015-9316-2

Publisher: Springer US

Persistent URL: http://hdl.handle.net/1721.1/104066

Version: Author's final manuscript: final author's manuscript post peer review, without publisher's formatting or copy editing

Terms of use: Creative Commons Attribution-Noncommercial-Share Alike 


\section{Grain boundary segregation in Al-Mn electrodeposits prepared from ionic liquid}

Contact information:

Ting-Yun Huang ${ }^{\mathrm{a}}$ : $\underline{\text { tingyun @ mit.edu }}$

Christopher J. Marvel'b cjm312@lehigh.edu

Patrick R Cantwell ${ }^{c}$ : cantwep@rose-hulman.edu

Martin P. Harmer ${ }^{\text {b. mph2@lehigh.edu }}$

Christopher A Schuh': schuh@mit.edu, 617-253-6910*Corresponding Author

Affiliation:

a. Departments of Materials Science and Engineering, Massachusetts Institute of Technology, 77 Massachusetts Avenue, Cambridge, MA, 02139, USA

b. Department of Materials Science and Engineering, Lehigh University, 27 Memorial Dr W, Bethlehem, PA, 18015, USA

c. Department of Mechanical Engineering, Rose-Hulman Institute of Technology, 5500 Wabash Ave, Terre Haute, IN 47803, USA 


\begin{abstract}
-200 words
Among the various preparation methods for nanocrystalline alloys, ionic liquid electrodeposition at low temperature is of interest for its scalability and efficiency. To achieve nanostructures with stabilized structures, it is desirable to directly deposit alloys in which the grain boundaries are decorated with a segregated alloying element. Here a combination of atom probe tomography and aberration corrected scanning transmission electron microscopy are used to confirm that in Al-Mn nanocrystalline alloys deposited from an ionic liquid, Mn is slightly segregated at grain boundaries in the as-deposited condition. The apparent heat of grain boundary segregation is calculated to lie between 1100 and $1500 \mathrm{~J} \mathrm{~mol}^{-1}$, which aligns reasonably well with a value calculated using a Miedema-based segregation model, and which is also in line with a more refined CALPHAD-type estimation if it is assumed that the Al-Mn deposits are not fully equilibrated at the deposition temperature.
\end{abstract}

\title{
Key words:
}

Nanocrystalline alloy, grain boundary segregation energy, electrodeposition, equilibrium 


\section{Introduction}

The wide degree of control over nucleation and growth kinetics available in electrodeposition can produce materials with a variety of internal structures, with grain sizes ranging from the micro- to the nanoscale[1-8]. Among them, nanocrystalline electrodeposits are particularly interesting due to the improvements in properties such as strength and hardness that result from grain refinement[9], and the ability of this technique to modulate composition and structure to nanometer scales[10]. However, because of their high grain boundary area densities, pure nanostructured metals generally coarsen rapidly even at low temperatures. As a result, there is increasing attention on alloys produced from electrodeposition baths, as the addition of solute elements to a nanocrystalline deposit can result in kinetic solute drag, which slows grain boundary motion, as well as thermodynamic energy reduction caused by segregation of solute atoms to grain boundaries[11-13].

Grain boundary segregation has been studied in a variety of electrodeposits, some of which are alloyed intentionally with the segregating species; systems such as Ni-W, Ni-P, Co-P, Co-W, and $\mathrm{Cu}-\mathrm{Bi}$ exhibit grain boundary segregation in the as-deposited state[14-18]. On the other hand, in some electrodeposits the minority elements are merely incorporated as an unintended impurity such as sulfur in ultrafine-grained Ni[19], and sulfur and carbon in Ni-Fe alloys[20]. Whether the segregating solute species is added intentionally or not, grain boundary segregation in all these alloys has proven beneficial in stabilization of the nanostructure.

$\mathrm{Ni}-\mathrm{P}$ is one of the more well-studied of these systems, in which Boylan et al.[21] found enhanced thermal stability up to $350^{\circ} \mathrm{C}$ using in-situ transmission electron microscopy (TEM). Further experiments were done by Hentschel et al. with atom-probe field-ion microscopy, which showed that in a nanocrystalline electroless Ni-3.6 at.\% P deposit, $\mathrm{P}$ is segregated at the grain boundaries with a local concentration as high as 11 at.\% in the as-deposited state[17]. Färber et al. further studied segregation of $\mathrm{P}$ at grain boundaries after heat treatment; the resulting $\mathrm{Ni}_{3} \mathrm{P}$ precipitates located at grain boundaries and boundary junctions, as well as a continuous $\mathrm{P}$ segregation zone, led to high thermal stability[22]. In the related Co-P system, Choi et al. reported P segregation leading to enhanced thermal stability by field ion microscopy[14]. In electrodeposited Ni-W, grain boundary segregation of $\mathrm{W}$ is sufficiently energetically important to the alloy thermodynamics that the $\mathrm{W}$ incorporation level directly controls the grain size that is produced 
upon deposition[23, 24], while also stabilizing the structure against grain growth[25]. For alloy systems like $\mathrm{Ni}-\mathrm{Cu}$ and $\mathrm{Ni}-\mathrm{Mn}$, the solute segregates to grain boundaries apparently only after exposure to elevated temperature[26,27]. Talin et al. showed that $\mathrm{Ni}$ deposits exhibited grain growth for annealing temperature between 200 and $300^{\circ} \mathrm{C}$, but Mn-containing Ni alloy deposits were able to retain strength and fine-grained structures up to $500^{\circ} \mathrm{C}$.

The above investigations that explore grain boundary segregation in electrodeposits focus on alloys synthesized from aqueous solutions. In light of the increasing effort devoted to electrodeposition from non-aqueous baths, in particular ionic liquid baths, it is of interest to understand whether similar alloy nanostructures can be produced from those media. Ionic liquid deposition has received considerable attention due to the low volatility, low flammability, and low toxicity of the electrolyte, as well as the wide electrochemical window properties seen in some systems[28-35]. These unique properties enable the deposition of low reduction potential metals such as aluminum, magnesium and titanium at moderate temperatures, or even semiconductors and compounds, $[36,37]$ without concern for hydrogen evolution. The technique can produce fully dense and deployable materials in the as-deposited condition if properly controlled [38, 39], and the internal structure can be finely tuned, by, e.g., controlling the applied current waveform[4,40]. Meanwhile, challenges such as a multiplicity of redox states[37] as well as insufficient adhesion at some substrate sites[41], and solution sensitivity to water[36] all need more attention.

With such technological benefits, it is clearly of interest whether nanostructured deposits produced from ionic liquids can be stabilized by grain boundary segregation of incorporated second species, in much the same way that similar deposits produced from aqueous solutions are. Yet, we are aware of no ionic liquid-derived electrodeposit that has been shown to exhibit grain boundary segregation in the as-deposited state, nor of any focused study of this issue.

In this work, we explore grain boundary segregation in aluminum-manganese alloys produced from an ionic liquid electrolyte. The binary Al-Mn system has a wide variety of equilibrium and metastable phases including solid solutions, several intermetallic phases, and a quasicrystalline icosahedral structure[42, 43]. Previous work from our group showed that Al-Mn alloys deposited from acidic chloroaluminate ionic liquid can produce many of these structures with a variety of characteristic length scales, ranging from microcrystalline to nanocrystalline, as well as dual- 
phase nanocrystalline-amorphous structures[4, 44]. What is more, for a duplex deposit containing both crystalline and amorphous regions, a three-dimensional atom probe tomography study of this system showed a subtle preference of Mn for the amorphous phase vis-à-vis the crystalline one[45]. Such a preference is an indication that Mn may perhaps also prefer the disordered regions in grain boundaries, however no grain boundaries were studied in that work and the possibility of Mn segregation to grain boundaries remains speculative[45]. In this paper we address this issue, and explicitly demonstrate that as-deposited Al-Mn alloys exhibit Mn segregation at the grain boundaries.

\section{Experimental procedure}

Al-Mn nanocrystalline alloys were prepared by electrodeposition from a chloroaluminate ionic liquid solution (1-ethyl-3-methylimidazolium and anhydrous aluminum chloride $\left(\mathrm{AlCl}_{3}\right)$ with a 2:1 molar ratio) in a nitrogen glovebox with water and oxygen content below $1 \mathrm{ppm}$, as described in more detail in Ref. [4]. Anhydrous manganese chloride ( $\mathrm{MnCl}_{2}, 99.99 \%$, Alfa Aesar) was added into the ionic liquid and stirred until it was fully dissolved with a concentration of $0.065 \mathrm{~mol} / \mathrm{L}$. Pure aluminum foil (99.99\% purity, purchased from Alfa Aesar) was used as the anode and polished copper (99\% purity) was used as the cathode. Pulse plating was conducted with a current waveform composed of $6 \mathrm{~mA} / \mathrm{cm}^{2}$ of cathodic pulse and $3 \mathrm{~mA} / \mathrm{cm}^{2}$ of anodic pulse, each of $20 \mathrm{~ms}$ duration, following the procedure in Ref. [40]. The total deposition time was 8 hours, leading to a deposit with a thickness around $10 \mu \mathrm{m}$. The sample was released by dissolving the copper substrate in concentrated nitric acid.

The deposit composition was measured using a JSM-6610LV scanning electron microscope (SEM) with calibrated energy-dispersive X-ray spectroscopy (EDS). X-ray diffraction (XRD) was carried out with a PANalytical X'Pert X-ray diffractometer operated at $45 \mathrm{kV}$ and $40 \mathrm{~mA}$ with a $\mathrm{Cu} \mathrm{K \alpha}$ source. The data was analyzed using Rietveld refinement to ascertain grain size and crystallinity. Transmission electron microscopy (TEM) was employed to study the grain size with a JEOL 2010F TEM, on samples prepared by electro-jet-polishing with 5\% perchloric acid in $20 \%$ butoxyethanol and $75 \%$ methanol under a voltage of $15 \mathrm{~V}$ at $-20^{\circ} \mathrm{C}$, using a Fischione model 100 twin-jet electropolisher. Scanning transmission electron microscopy (STEM) samples 
were prepared via the in-situ lift-out method in a FEI DB235 focused ion beam (FIB) and final polishing was completed to remove the amorphized damage layer in a Fischione 1040 NanoMill. STEM analysis was conducted in a JEOL JEM-ARM200CF at 200kV using bright field (BF) and high-angle annular dark field (HAADF) imaging. EDS line scans were performed with a JEOL 100- $\mathrm{mm}^{2} \mathrm{x}$-ray detector. Prior to analysis, an SPI plasma cleaner was employed to remove excess carbon from the sample surface.

A variety of atom probe samples were prepared by FIB lift-out with a combination of pyramid milling and annular milling described in [46-50] to reduce the Ga implantation. The lift-out procedure was conducted with a FEI Helios FIB operated at 30, 16, 8, and a $5 \mathrm{kV}$ final cleaning step to remove the amorphous layer. The sample tip diameters ranged from 50 to $80 \mathrm{~nm}$. Tips were observed with the JEOL 2100 TEM to study the tip geometry and structure for better reconstruction.

The atom probe tips were analyzed using a CAMECA LEAP 4000X HR instrument operated under voltage mode with a pulse fraction ranging from 15 to $18 \%$ and pulse repetition rate of 200 $\mathrm{kHz}$ at $50 \mathrm{~K}$. The datasets acquired by atom probe ranged from 3 to 34.5 million atoms. Data analysis was conducted using IVAS software and the results were output for further analysis by custom algorithms.

To test the distribution of the Mn solute inside the Al-Mn deposits using APT data, binomial fitting was used. If a sample were a perfectly random solution, the frequency distribution of the composition when measured many times in small blocks would be binomial.[51] We divided individual samples into many blocks with $\mathrm{n}=200$ atoms inside each to obtain the composition distribution. The binomial distribution describes a probability density $P(c)$ at concentration $c$ as 
$P(c)=\frac{1}{\sigma \sqrt{2 \pi}} \exp \left(\frac{-\left(c-c_{0}\right)^{2}}{2 \sigma^{2}}\right)$

where $c_{0}$ is the average concentration the whole sample, $c$ is the concentration for individual blocks, and the width normalization is given by $\sigma^{2}=c_{0}\left(1-c_{0}\right) / n$. The binomial equation involves only a single fitting parameter, namely the average solute concentration; deviation from a single-variable fit suggests a nonrandom solute distribution.

\section{Results}

\section{Basic structure characterization}

The average concentration of the Al-Mn deposit is measured as $6.8( \pm 0.4)$ at.\% Mn by SEM EDS. The XRD pattern and TEM micrograph in Figure 1 confirm the presence of face-centered cubic crystalline Al phase, with no evidence of any additional phases, which is in line with prior references [4]. Using a combination of dark-field and bright-field imaging of over 100 grains, the average grain size was measured as $162 \mathrm{~nm}$. The inset selected area diffraction (SAD) pattern in Figure 1(b) confirms a fine-grained FCC structure with no evidence for additional phases.

\section{Aberration-corrected STEM results}

To examine the grain boundary segregation behavior in the Al-Mn system, we characterized the grain boundary structure and obtained chemical signatures from the grain boundary and grain interiors. The BF and HAADF images seen in Figure 2 demonstrate the typical appearance of two adjacent grains. The increased intensity via HAADF along two boundaries, seen in Figure 2(b), suggests there is a local increase of average atomic number. Thus, in this alloy, the increased intensity is likely Mn segregation. Unfortunately, Mn-enrichment is typically not as evident at higher magnifications, as seen in Figure 2(c) and (d).

A concentration profile across another such grain boundary is shown in Figure 3. The Mn and Al concentrations, calculated to $2 \sigma$ error, are determined using the Cliff-Lorimer equation, and the $\mathrm{K}$ factor is determined by substituting the global concentration as the grain interior concentration. The Mn content reaches a maximum of approximately 14 at. $\%$ at the grain boundary, as compared to $\sim 7-8$ at.\% in the grains, and confirms that the high intensity in HAADF is Mn segregation. 
Generally, EDS line scans do not provide absolute interfacial compositions because of grain boundary inclination. To more accurately measure the Mn-enrichment, and eliminate the effect of boundary inclination, the scanwidth method [52] was used to determine extent of $\mathrm{Mn}$ segregation via Eq. (2)

$M L_{S_{A}}=N_{B}^{(1 / 3)} \times \frac{I_{\mathrm{A}}}{I_{\mathrm{B}}} \times w \times k_{\mathrm{AB}}$

where $\boldsymbol{M} \boldsymbol{L}_{\boldsymbol{S}_{A}}$ is the number of Mn solute atoms per monolayer, $\boldsymbol{N}_{\mathrm{B}}$ is the atom number density of the Al matrix, $\boldsymbol{I}_{\mathrm{A}}$ and $\boldsymbol{I}_{\mathrm{B}}$ are the EDS intensities of the Mn and Al, and $\boldsymbol{w}$ is the EDS scan width perpendicular to the grain boundary[52]. $\boldsymbol{N}_{B}^{(2 / 3)}$ is determined by taking the area density of the most densely packed plane, which is $\{111\}$ for FCC Al. Eq. (1) gives a Mn area density of $4.2 \pm 0.2$ atoms $/ \mathrm{nm}^{2}$ and $2.6 \pm 0.1$ atoms $/ \mathrm{nm}^{2}$ on the grain boundary and grain interiors, respectively. Thus, the difference of approximately 1.6 atoms $/ \mathrm{nm}^{2}$ represents the excess of $\mathrm{Mn}$ on the grain boundary. Because the sample thickness is unknown, an effective scan width was not utilized to eliminate the effect of beam broadening. The line profile and scanwidth results are in good agreement as both techniques estimate $~ 50-60 \%$ enrichment of Mn on the boundary.

In addition to the results seen in Figure 2 and Figure 3, we surveyed several more individual grain boundaries; grain boundary segregation is clearly observed in most but not all of these. In light of the broad spectrum of different grain boundary characters that might be sampled in a polycrystal, it is reasonable that a variety of different responses are observed. We will comment further on this later.

\section{APT results}

Results from atom probe tomography also suggest a tendency for grain boundary segregation of solute. An atom probe tip and the corresponding reconstruction atom dot maps are shown in Figure 4(a) to (c), where several grain boundaries that appear to span the sample are captured. A line scan with a block size of 1.8 by $1.8 \mathrm{~nm}^{2}$ along the axial direction (z direction) was carried out. Mn concentrations along this direction which exceeded two standard deviation of the average concentration are marked; their positions are indicated by green arrows in Figure 4(b)). Their corresponding locations are also marked in the TEM image in Figure 4(a). The Ga distribution (Figure 4(c), blue dots) also aligns with the Mn distribution and coincides with the grain boundary positions in the TEM micrograph; as suggested in Ref. [45], Ga is an effective marker 
of grain boundaries in APT of aluminum. Taken together, the above results positively identify several individual grain boundaries, and confirm than $\mathrm{Mn}$ is located preferentially at them.

Knowing the positions of the grain boundaries, we can proceed to conduct quantitative analysis using ladder diagrams as shown in Figure 5, which includes several parallel profiles across the same grain boundary at different positions, each 1.8 by $1.8 \mathrm{~nm}$ in cross section. A ladder diagram is a cumulative presentation of solute content along a one-dimensional line, plotting the number of solute atoms encountered as a function of the total number of atoms encountered. Such ladder diagrams have been extensively used for identifying different phases as well as clustering in APT data.[23, 53, 54] The slopes of these ladder diagrams are indicated in Figure 5, and represent the local concentration; in every case there is a clear signal of Mn segregation at the grain boundary, and the amount of grain boundary segregation ranges from 3 to 6 at.\% higher than the composition in the neighboring grains.

To get better statistics on the grain boundary and grain compositions, we constructed and analyzed 180 ladder diagrams such as those of Figure 5, to assemble composition distributions unique to the boundary or the grains. The result is shown in Figure 6. Figure 6(a) shows a histogram of Mn concentrations at the grain boundary and Figure 6(b) shows a histogram in the grain interiors; the grain boundary is indeed enriched in Mn. Figure 6(c) shows a cumulative probability plot of the difference between grain boundary and grain interior sites on individual ladder diagrams, which suggests a median enrichment of Mn at boundaries of about 3 at.\%.

These subtle Mn increases at the grain boundaries can also be verified indirectly using binomial compositional analysis on entire APT samples. We divide the sample into multiple blocks with $\mathrm{n}$ $=200$ atoms inside to obtain the composition distribution in Figure 7(a), and by treating $c_{0}$ as the sole fitting parameter, Eq. (1) can be fitted to the experimental data of Figure 7(a) as shown in red. The fit is significantly different from the shape of the data, which exhibits skew to higher concentrations. Such skew is what would be expected if there were a minority of sites of higherthan-average composition, i.e., if there were selective segregation of solute to some regions in the sample (grain boundaries). 
Since the sample may therefore better be described as a combination of two different regions (crystals and grain boundaries), each of which is a random solution at its own composition, we further fitted the data with a two-binomial distribution as

$P(c)=\frac{f_{g i}}{\sigma_{g i} \sqrt{2 \pi}} \exp \left(\frac{-\left(c-c_{g i}\right)^{2}}{2 \sigma_{g i}^{2}}\right)+\frac{f_{g b}}{\sigma_{g b} \sqrt{2 \pi}} \exp \left(\frac{-\left(c-c_{g b}\right)^{2}}{2 \sigma_{g b}^{2}}\right)$

in which the subscript $g_{i}$ denotes the grain interior and $g_{b}$ denotes the grain boundary regions, each present in their own volume fraction, $f$ and at separate compositions, c.

The binomial-binomial fit of Eq. (3) to the data of Figure 7(a) is also shown in Figure 7(a), along with the sub-distributions corresponding to each region. The fit reveals an average grain interior concentration of 7.5 at.\% (dotted blue line) and an average grain boundary concentration of 12.6 at.\% (dashed blue line). The grain interior concentration is within the error of the bulk $\mathrm{Mn}$ concentration measured by SEM-EDS, and the enhancement at the boundary of roughly 5 at $\%$ is reasonably aligned with the results of Figure 6 . The grain boundary fraction fitted in the binomial fitting is $12 \%$ of the total volume, which aligns reasonably well with a geometric estimate based on isoconcentration surfaces such as shown in Figure 7(b), which also suggests that grain boundaries comprise $\sim 7-10 \%$ of the sample. Note that in Figure $7(\mathrm{~b})$, the anomalous region at the top of the analyzed volume is associated with Ga damage near an exposed crystallographic pole, however, this region represents less than $2 \%$ of the total analysis volume.

Interestingly, the distributions of grain boundary concentration and grain interior concentration in Figure 6 exhibit some overlap, which allows for the possibility of individual grain boundaries exhibiting little or no solute segregation. This aligns with the STEM observations described above, where some boundaries but not all exhibited compositional contrast. Since grain boundary segregation is known to depend on the misorientation and boundary plane indices [55], it is reasonable that a survey of a variety of boundaries such as we have conducted here would reveal a spectrum of responses. 


\section{Discussion}

The above characterizations are all consistent with one another, and confirm that the as-deposited Al-Mn alloy in this work is a single-phase FCC polycrystal solid solution with a fine grain structure, and with subtle but clear enhancement of Mn at the grain boundaries of $\sim 3-5 \%$ as compared to the bulk concentration. While there are many different types of grain boundaries, each with different segregation behavior, and also different sites within any individual boundary, these results speak to the average, or effective grain boundary segregation behavior. Grain boundary segregation results from a positive heat of grain boundary segregation of the solute species, i.e., it is a reflection of an energetic preference for solute to occupy grain boundary sites. Were the system assumed to be a solid solution in equilibrium (a significant assumption for electrodeposition, which we will test shortly), an effective heat of segregation $\left(H_{\text {seg }}\right)$ can be estimated based on the quantitative STEM and APT composition measurements using the McLean segregation isotherm:

$\frac{C_{g b}}{1-C_{g b}}=\frac{C_{g i}}{1-C_{g i}} \exp \left(\frac{H_{s e g}}{k T}\right)$

Where the composition subscript gi denotes the grain interior and $g b$ denotes the grain boundary. Taking the relevant temperature to be the processing temperature $\mathrm{T}=300 \mathrm{~K}$, the $\mathrm{H}_{\text {seg }}$ values obtained from the experimental results are shown in Table 1, and range from 1100 to $1500 \mathrm{~J} \mathrm{~mol}^{-}$ 1. Such values are very low, suggestive of a weak segregation tendency, which is of course in line with the subtle composition inflation we measured.

Reliable grain boundary segregation energies are not generally available for arbitrary alloy pairs, and for the Al-Mn system our best point of comparison with theory is with a Miedema-based model for the grain boundary segregation energy. The calculation was proposed by Murdoch and $\operatorname{Schuh}[56]$, and gives the dilute limit grain boundary segregation enthalpy $\left(\Delta H_{0, \mathrm{M}}^{\mathrm{Seg}}\right)$

$\Delta H_{0, M}^{S e g}=-0.71 \times \frac{1}{3} \times v \times\left[-\Delta H_{B i n A}^{i n t}-c_{M} \gamma_{A}^{S} V_{A}^{2 / 3}+c_{M} \gamma_{B}^{S} V_{B}^{2 / 3}\right]+\Delta E_{e l}$

in which the subscripts B denotes solute and A solvent, $\boldsymbol{\Delta} \boldsymbol{H}_{\mathrm{BinA}}^{\mathrm{int}}$ is the interaction energy (i.e., the bond-level heat of mixing) for a B atom surrounded by A atoms, $\boldsymbol{c}_{\boldsymbol{M}}$ is a dimensionless semiempirical constant equal to $\mathbf{4 . 5} \times \mathbf{1 0}^{\mathbf{8}}, \boldsymbol{\gamma}^{\boldsymbol{S}}$ is the surface energy for the pure subscripted metal, $\mathrm{V}$ is the atomic volume, and $\boldsymbol{v}=\mathbf{1 / 2}$ describes the fraction of bonds lost at grain boundaries [57]. 
The elastic term is based on an Eshelby-type continuum linear elastic formalism[58, 59] for the energy of substituting a misfitting solute atom inside the solvent matrix:

$\Delta E_{e l}=\frac{24 \pi K_{A} G_{B} r_{B} r_{A}\left(r_{A}-r_{B}\right)^{2}}{3 K_{A} r_{A}+4 G_{B} r_{B}}$

Here, $\mathrm{K}$ is the bulk modulus, $\mathrm{G}$ is the shear modulus, and $\mathrm{r}$ is the atomic radius.

With this equation and numerical values from Miedema[60], de Boer[61] and James et al.[62] (which are all assembled in Table 2), the expected grain boundary segregation energy for Mn in Al can be calculated using equation (6). To keep the spirit of a Miedema-type estimate, we use here Miedema's original estimate of the system heat of mixing for Mn dissolved in Al $\Delta H_{\mathrm{BinA}}^{\mathrm{int}}=$ $-106 \mathrm{~kJ} \mathrm{~mol}^{-1}$ [60]. The calculated Mn grain boundary segregation energy is $1030 \mathrm{~J} \mathrm{~mol}^{-1}$. This value is surprisingly well aligned with the experimental data based on an assumed equilibrium state in the deposit $\left(H_{\mathrm{seg}} \sim 1100-1500 \mathrm{~J} \mathrm{~mol}^{-1}\right)$ both in its positive sign (i.e., grain boundary segregation is favored) and its magnitude.

One possible interpretation of this close alignment is that the deposition process used to make this alloy is sufficiently close to equilibrium growth that it is able to find a near-equilibrium condition and grow a grain structure with the preferred grain boundary solute content. This is at least somewhat plausible in light of the kinetic calculation of Ruan and Schuh[4], who estimated that the average atom might diffuse around $20 \mathrm{~nm}$ over the surface before becoming buried in an Al-Mn electodeposit, and for our deposition conditions a somewhat larger diffusion length of perhaps $40 \mathrm{~nm}$ is kinetically reasonable. In this scenario, the majority of atoms would experience both bulk and grain boundary states as they traverse the surface during deposition, and might therefore preferentially assemble into a near-equilibrium grain boundary segregation state.

In this scenario, the grain boundary segregated state is of lower energy than the competing solid solution, but it is an interesting question whether it would successfully compete with the equilibrium diagram that involves intermetallic $\mathrm{Al}_{6} \mathrm{Mn}$. In nanocrystalline materials, it has been suggested that grain boundary segregated polycrystals can energetically compete with second phases in equilibrium[63]. In similar Al-Mn electrodeposits studied in prior work, heating has 
been shown to lead to intermetallic formation[4, 64-67], which suggests that the polycrystalline state may be out of equilibrium, as discussed next.

The second possibility is that the deposit is not in equilibrium, but rather is in a kinetically trapped condition, and the grain boundary segregation we observe is therefore a signature of thermodynamic preference but is not equilibrated. If the polycrystalline state is already viewed as a metastable state excited above an intermetallic ground state, then incomplete grain boundary segregation would represent an even further degree of excitation (i.e., higher-energy metastable condition entrapped kinetically). In this case the true grain boundary segregation energy would be higher, and underestimated by the above model. There is some reason to expect this situation. For example, the Miedema heat of mixing for $\mathrm{Mn}$ in $\mathrm{Al}$ used above $\left(\Delta H_{\mathrm{BinA}}^{\mathrm{int}}=-106 \mathrm{~kJ} \mathrm{~mol}^{-1}[60]\right)$ is somewhat higher than one obtains from an analysis of CALPHAD-type free energy functions for the Al-Mn system which are used to generate the phase diagram[42]. Using such equations in the dilute limit of Mn concentration yields an alternative value of $\Delta H_{\mathrm{BinA}}^{\mathrm{int}}=-70 \mathrm{~kJ} \mathrm{~mol}^{-1}$. Using this value to correct the estimate of equation (5) yields a somewhat higher grain boundary segregation energy of $5300 \mathrm{~J} \mathrm{~mol}^{-1}$. If this value is more reflective of the true grain boundary segregation energy for $\mathrm{Mn}$ in $\mathrm{Al}$, then the fact that it is four to five times higher than the effective value extracted from the experimental results would align with the electrodeposit being out of equilibrium, i.e. not fully segregated to the extent it could be. Future work examining changes in segregation state upon heating might prove helpful in more precisely determining the true value of the grain boundary segregation energy.

\section{Conclusion}

The grain boundary segregation behavior of electrodeposited Al-6.7 at.\% Mn has been studied experimentally using aberration-corrected STEM and APT, and compared with a Miedemabased grain boundary segregation model. Both the STEM and APT results revealed that Mn is segregated to grain boundaries in the as-deposited condition, and showed reasonable quantitative agreement with one another. Using the McLean isotherm (and therefore assuming an equilibrium condition) gives a grain boundary segregation energy of order 1100-1500 J mol-1. This very low energy level suggests that segregation is either very subtly favored 
thermodynamically in this system, or the deposit is out of equilibrium and does not find as segregated a condition as it would like. Interestingly, both of these possibilities are supported by Miedema-type calculations; a simple calculation yielded roughly $1000 \mathrm{~J} \mathrm{~mol}^{-1}$ using the classical Miedema interaction energy for $\mathrm{Al}$ and $\mathrm{Mn}$, while the use of a more experimentally relevant interaction energy based on the calculated bulk phase diagram gives $5030 \mathrm{~J} \mathrm{~mol}^{-1}$. The first of these calculated values matches the measurements, while the second would anticipate greater segregation if the sample were able to fully equilibrate.

\section{Acknowledgement}

This research was supported (in part) by the U.S. Army Research Office under contract W911NF-13-D-0001 through the Institute of Soldier Nanotechnologies at MIT. The APT work was performed in part at the Center for Nanoscale Systems (CNS), a member of the National Nanotechnology Infrastructure Network (NNIN), which is supported by the National Science Foundation under NSF award no. ECS-0335765. CNS is part of Harvard University. The aberration corrected STEM work was supported with ONR-MURI program, grant number N00014-11-0678. 


\section{Reference}

1. Qu N., Zhu D, Chan K., Lei W. (2003) Pulse electrodeposition of nanocrystalline nickel using ultra narrow pulse width and high peak current density. Surf Coatings Technol 168:123-128. doi: $10.1016 / \mathrm{S} 0257-8972(03) 00014-8$

2. Natter H, Hempelmann R (2008) Nanocrystalline Metals Prepared by Electrodeposition. Zeitschrift für Phys Chemie 222:319-354. doi: 10.1524/zpch.2008.222.2-3.319

3. Bicelli LP, Bozzini B, Mele C, D’Urzo L (2008) A review of nanostructural aspects of metal electrodeposition. Int J Electrochem Sci 3:356-408.

4. Ruan S, Schuh CA (2009) Electrodeposited Al-Mn alloys with microcrystalline, nanocrystalline, amorphous and nano-quasicrystalline structures. Acta Mater 57:3810-3822. doi: 10.1016/j.actamat.2009.04.030

5. Stafford GR (1989) The Electrodeposition of an Aluminum-Manganese Metallic Glass from Molten Salts. J Electrochem Soc 136:635. doi: 10.1149/1.2096701

6. Nakanishi T, Ozaki M, Nam H-S, et al. (2001) Pulsed Electrodeposition of Nanocrystalline CoNiFe Soft Magnetic Thin Films. J Electrochem Soc 148:C627. doi: 10.1149/1.1388886

7. Tsuda T, Hussey CL, Stafford GR (2005) Electrodeposition of Al-Mo-Mn Ternary Alloys from the Lewis Acidic AlCl[sub 3]-EtMeImCl Molten Salt. J Electrochem Soc 152:C620. doi: $10.1149 / 1.1995696$

8. Mishra AC, Thakur AK, Srinivas V (2009) Effect of deposition parameters on microstructure of electrodeposited nickel thin films. J Mater Sci 44:3520-3527. doi: 10.1007/s10853-009$3475-\mathrm{y}$

9. Nieh TG, Wadsworth J (1991) Hall-Petch relation in nanocrystalline solids. Scr Metall Mater 25:955-958.

10. Yahalom J, Zadok O (1987) Formation of composition-modulated alloys by electrodeposition. J Mater Sci 22:499-503. doi: 10.1007/BF01160760

11. Koch CC, Scattergood RO, Darling K a., Semones JE (2008) Stabilization of nanocrystalline grain sizes by solute additions. J Mater Sci 43:7264-7272. doi: 10.1007/s10853-008-2870-0

12. Weissmiiller J (1993) Alloy Effects in Nanostructures. Nanostructured Mater 3:261-272.

13. Liu F, Kirchheim R (2004) Grain boundary saturation and grain growth. Scr Mater 51:521525. doi: 10.1016/j.scriptamat.2004.05.042 
14. Choi P, Dasilva M, Klement U, et al. (2005) Thermal stability of electrodeposited nanocrystalline Co-1.1at.\%P. Acta Mater 53:4473-4481. doi:

10.1016/j.actamat.2005.06.006

15. Chen X, Mao J (2010) Thermal Stability and Tensile Properties of Electrodeposited Cu-Bi Alloy. J Mater Eng Perform 20:481-486. doi: 10.1007/s11665-010-9700-7

16. Detor AJ, Miller MK, Schuh CA (2006) Solute distribution in nanocrystalline Ni-W alloys examined through atom probe tomography. Philos Mag 86:4459-4475. doi:

$10.1080 / 14786430600726749$

17. Hentschel T, Isheim D, Kirchheim R (2000) Nanocrystalline Ni-3.6 at.\% P and its transformation sequence studied by atom-probe field-ion microscopy. Acta Mater 48:933941.

18. Weston DP, Gill SPA, Fay M, et al. (2013) Nano-structure of Co-W alloy electrodeposited from gluconate bath. Surf Coatings Technol 236:75-83. doi: 10.1016/j.surfcoat.2013.09.031

19. McFadden SX, Mukherjee a. K (2005) Sulfur and superplasticity in electrodeposited ultrafine-grained Ni. Mater Sci Eng A 395:265-268. doi: 10.1016/j.msea.2004.12.025

20. Liu Y, Liu L, Shen B, Hu W (2011) A study of thermal stability in electrodeposited nanocrystalline Fe-Ni invar alloy. Mater Sci Eng A 528:5701-5705. doi:

10.1016/j.msea.2011.04.052

21. Boylan K, Ostrander D, Erb U, et al. (1991) An in-situ TEM study of the thermal stability of nanocrystalline Ni-P. Scr Metall Mater 25:2711-2716.

22. Färber B, Cadel E, Menand A, et al. (2000) Phosphorus segregation in nanocrystalline Ni-3.6 at.\% $\mathrm{P}$ alloy investigated with the tomographic atom probe (TAP). Acta Mater 48:789-796.

23. Detor AJ, Miller MK, Schuh CA (2007) Measuring grain-boundary segregation in nanocrystalline alloys: direct validation of statistical techniques using atom probe tomography. Philos Mag Lett 87:581-587. doi: 10.1080/09500830701400125

24. Detor AJ, Schuh C (2007) Grain boundary segregation, chemical ordering and stability of nanocrystalline alloys: Atomistic computer simulations in the $\mathrm{Ni}-\mathrm{W}$ system. Acta Mater 55:4221-4232. doi: 10.1016/j.actamat.2007.03.024

25. Detor AJ, Schuh CA (2007) Microstructural evolution during the heat treatment of nanocrystalline alloys. J Mater Res 22:3233-3248. doi: 10.1557/JMR.2007.0403

26. Talin AA, Marquis EA, Goods SH, et al. (2006) Thermal stability of Ni-Mn electrodeposits. Acta Mater 54:1935-1947. doi: 10.1016/j.actamat.2005.12.027 
27. Pellicer E, Varea A, Sivaraman KM, et al. (2011) Grain boundary segregation and interdiffusion effects in nickel-copper alloys: an effective means to improve the thermal stability of nanocrystalline nickel. ACS Appl Mater Interfaces 3:2265-74. doi: 10.1021/am2004587

28. Armand M, Endres F, MacFarlane DR, et al. (2009) Ionic-liquid materials for the electrochemical challenges of the future. Nat Mater 8:621-9. doi: 10.1038/nmat2448

29. Abedin SZ El, Polleth M, Meiss S a., et al. (2007) Ionic liquids as green electrolytes for the electrodeposition of nanomaterials. Green Chem 9:549. doi: 10.1039/b614520e

30. Tsuda T, Hussey CL (2007) Electrochemical Applications of Room-Temperature Ionic Liquids. Electrochem Soc Interface. doi: 10.1002/elps.200800561

31. Jiang T, Chollier Brym MJ, Dubé G, et al. (2006) Electrodeposition of aluminium from ionic liquids: Part I - electrodeposition and surface morphology of aluminium from aluminium chloride (AlCl3)-1-ethyl-3-methylimidazolium chloride ([EMIm]Cl) ionic liquids. Surf Coatings Technol 201:1-9. doi: 10.1016/j.surfcoat.2005.10.046

32. Jiang T, Chollier Brym MJ, Dubé G, et al. (2006) Electrodeposition of aluminium from ionic liquids: Part II - studies on the electrodeposition of aluminum from aluminum chloride (AICl3) - trimethylphenylammonium chloride (TMPAC) ionic liquids. Surf Coatings Technol 201:10-18. doi: 10.1016/j.surfcoat.2005.12.024

33. NuLi Y, Yang J, Wang J, et al. (2005) Electrochemical Magnesium Deposition and Dissolution with High Efficiency in Ionic Liquid. Electrochem Solid-State Lett 8:C166. doi: $10.1149 / 1.2052048$

34. Tsuda T, Hussey CL, Stafford GR, Bonevich JE (2003) Electrochemistry of Titanium and the Electrodeposition of Al-Ti Alloys in the Lewis Acidic Aluminum Chloride-1-Ethyl-3methylimidazolium Chloride Melt. J Electrochem Soc 150:C234. doi: 10.1149/1.1554915

35. Kong BO, Suk JI, Nam SW (1996) Identification of Mn-dispersoid in Al-Zn-Mg-Mn alloy. J Mater Sci Lett 15:763-766. doi: 10.1007/BF00274597

36. Abbott AP, Frisch G, Ryder KS (2013) Electroplating Using Ionic Liquids. Annu Rev Mater Res 43:335-358. doi: 10.1146/annurev-matsci-071312-121640

37. Armand M, Endres F, MacFarlane DR, et al. (2009) Ionic-liquid materials for the electrochemical challenges of the future. Nat Mater 8:621-629. doi: 10.1038/nmat2448

38. Clark D, Wood D, Erb U (1997) Industrial applications of electrodeposited nanocrystals. Nanostructured Mater 9:755-758. 
39. Wei Y (2004) Grain-boundary sliding and separation in polycrystalline metals: application to nanocrystalline fcc metals. J Mech Phys Solids 52:2587-2616. doi: 10.1016/j.jmps.2004.04.006

40. Ruan S, Schuh CA (2012) Towards electroformed nanostructured aluminum alloys with high strength and ductility. J Mater Res 27:1638-1651. doi: 10.1557/jmr.2012.105

41. Zein El Abedin S, Endres F (2013) Challenges in the electrochemical coating of highstrength steel screws by aluminum in an acidic ionic liquid composed of 1-Ethyl-3methylimidazolium chloride and AlCl3. J Solid State Electrochem 17:1127-1132. doi: 10.1007/s10008-012-1973-3

42. Du Y, Wang J, Zhao J, et al. (2007) Reassessment of the Al-Mn system and a thermodynamic description of the Al-Mg-Mn system. J Mater Res 855-871. doi: $10.3193 / 146.101547$

43. Shechtman D, Blech I (1984) Metallic Phae with Long-Range Orientational Order and No Translational Symmetry. Phys Rev Lett 53:1951-1954.

44. Cai W, Schuh CA (2012) Tuning nanoscale grain size distribution in multilayered Al-Mn alloys. Scr Mater 66:194-197. doi: 10.1016/j.scriptamat.2011.10.040

45. Ruan S, Torres KL, Thompson GB, Schuh CA (2011) Gallium-enhanced phase contrast in atom probe tomography of nanocrystalline and amorphous Al-Mn alloys. Ultramicroscopy 111:1062-72. doi: 10.1016/j.ultramic.2011.01.026

46. Felfer P (2012) Atom probe sample preparation using FIB: ref-guide/case studies.

47. Felfer PJ, Alam T, Ringer SP, Cairney JM (2012) A reproducible method for damage-free site-specific preparation of atom probe tips from interfaces. Microsc Res Tech 75:484-91. doi: $10.1002 /$ jemt.21081

48. Rachbauer R, Mass1 S, Stergar E, et al. (2010) Atom probe specimen preparation and 3D interfacial study of Ti-Al-N thin films. Surf Coatings Technol 204:1811-1816. doi: 10.1016/j.surfcoat.2009.11.020

49. Miller MK, Russell KF (2007) Atom probe specimen preparation with a dual beam SEM/FIB miller. Ultramicroscopy 107:761-6. doi: 10.1016/j.ultramic.2007.02.023

50. Miller MK, Russell KF, Thompson K, et al. (2007) Review of atom probe FIB-based specimen preparation methods. Microsc Microanal 13:428-36. doi: $10.1017 / \mathrm{S} 1431927607070845$

51. Cowan G (1998) Statistical Data Analysis. 
52. Alber U, Müllejans H, Rühle M (1997) Improved quantification of grain boundary segregation by EDS in a dedicated STEM. Ultramicroscopy 69:105-116.

53. Gault B, Moddy MP, Cairney JM, Ringer SP (2012) Atom Probe Microscopy. doi: 10.1007/978/-1-4614-3436-8

54. Mottura a, Miller MK, Reed RC (2008) Atom probe tomography analysis of possible rhenium clustering in nickel-based superalloys. Superalloys 2008 891-900. doi: 10.7449/2008/Superalloys_2008_891_900

55. Herbig M, Raabe D, Li YJ, et al. (2014) Atomic-Scale Quantification of Grain Boundary Segregation in Nanocrystalline Material. Phys Rev Lett 112:126103. doi: 10.1103/PhysRevLett.112.126103

56. Murdoch H a., Schuh C a. (2013) Stability of binary nanocrystalline alloys against grain growth and phase separation. Acta Mater 61:2121-2132. doi: 10.1016/j.actamat.2012.12.033

57. Trelewicz J, Schuh C (2009) Grain boundary segregation and thermodynamically stable binary nanocrystalline alloys. Phys Rev B 79:1-13. doi: 10.1103/PhysRevB.79.094112

58. Eshelby JD (1954) Distortion of a Crystal by Point Imperfections. J Appl Phys 25:255. doi: $10.1063 / 1.1721615$

59. Eshelby JD (1957) The determination of the elastic field of an ellipsoidal inclusion, and related problems. Proc. R. Soc. Phys. Sci. Res. J.

60. Bakker H (1998) Enthalpies in alloys- Miedema's semi-empirical model. Trans Tech Publications Ltd

61. De Boer FR, Boom R, Mattens WCM, et al. (1988) Cohesion in metals: transition metal alloys. North Holland

62. James AM, Loard MP (1992) Macmillan's Chemical and Physical Data. Macmillan, London, UK

63. Chookajorn T, Murdoch HA, Schuh CA (2012) Design of stable nanocrystalline alloys. Science 337:951-4. doi: 10.1126/science.1224737

64. Grushko B, Stafford GR (1989) Structural Study of Electrodeposited Aluminum-Manganese Alloys. Metall Trans A 20:1351-1359.

65. Stafford GR, Grushko B, McMichael RD (1993) The electrodeposition of Al-Mn ferromagnetic phase from molten salt electrolyte. J Alloys Compd 200:107-113. doi: $10.1016 / 0925-8388(93) 90479-7$ 
66. Grushko B, Stafford GR (1989) A structural study of a metastable phase in Al-Mn alloys electrodeposited from molten salts. Scr Metall Mater Metall Mater 23:557-562.

67. Grushko B, Stafford GR (1990) Phase Formation in Electrodeposited and Thermally Annealed Al-Mn Alloys. Metall Trans A 21:2869-2879. 


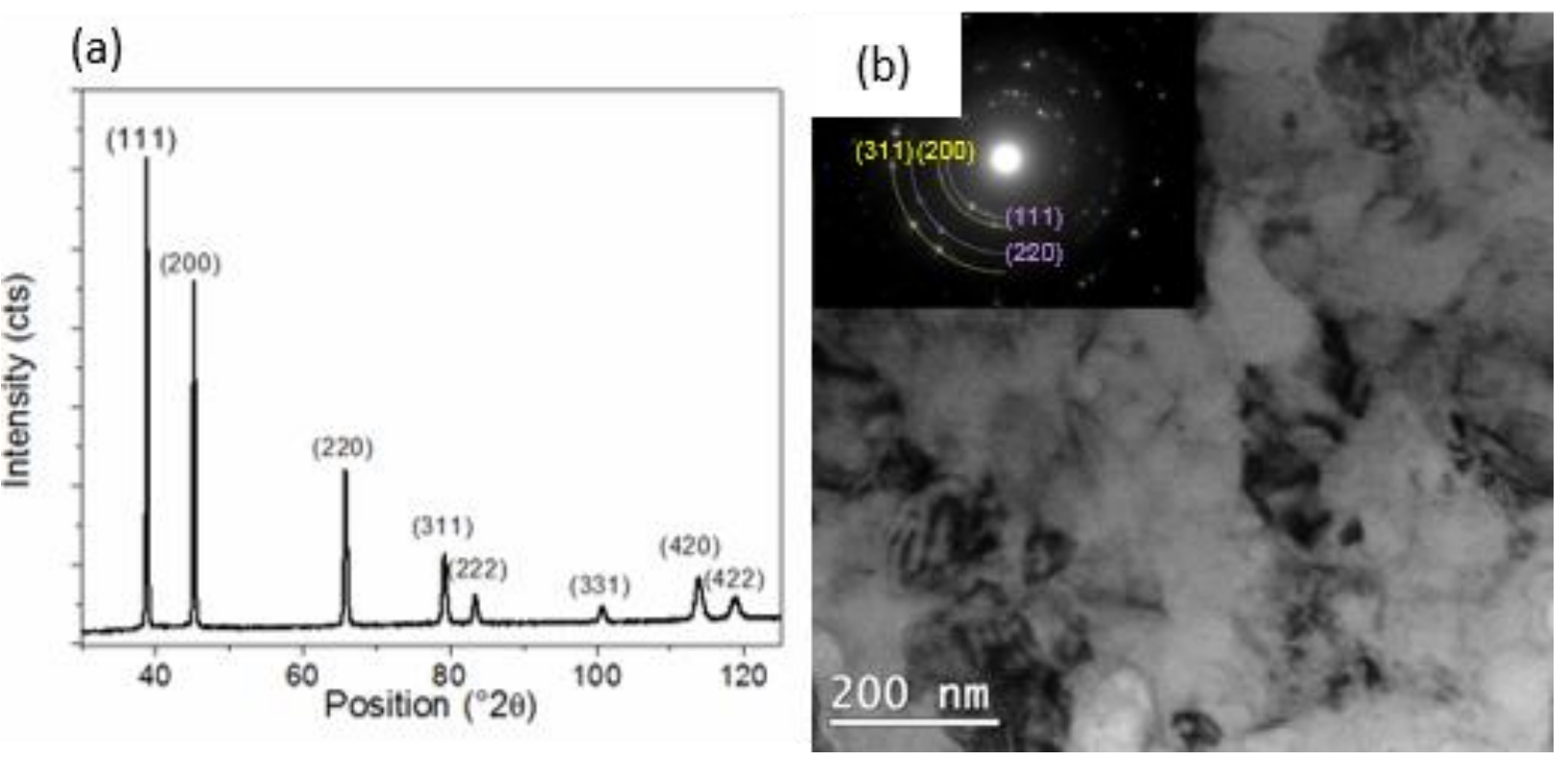

Figure 1 Structural characterization of the as-deposited Al-Mn alloy film by (a) XRD and (b) bright field TEM. (a) shows that the material is Al FCC crystalline phase. (b) shows the grain size (in plan view) is about $162 \mathrm{~nm}$ on average, and the inset SAD pattern conforms to a single phase FCC structure. 

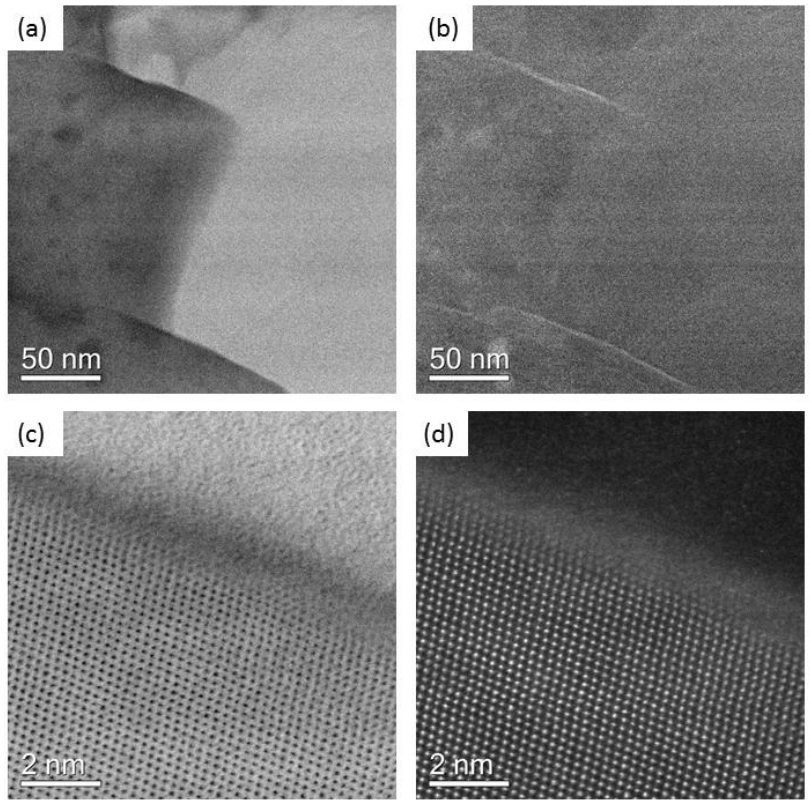

Figure 2 Images of typical grain boundaries under Cs-corrected STEM. The low magnification BF image (a) depicts two adjacent grains via and low magnification HAADF (b) shows the two grain boundaries exhibit Mn segregation. Images (c) and (d) are high magnification BF and HAADF, respectively. 


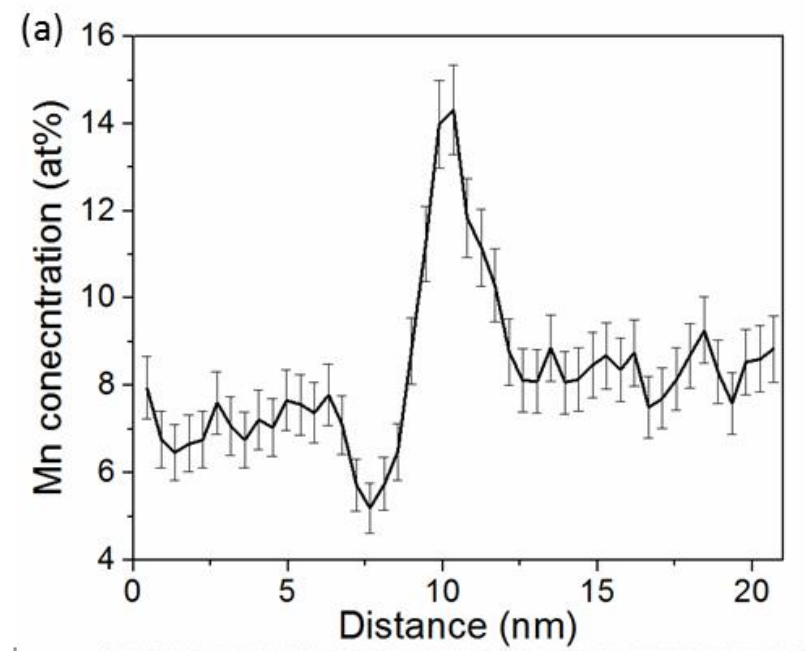

(b)

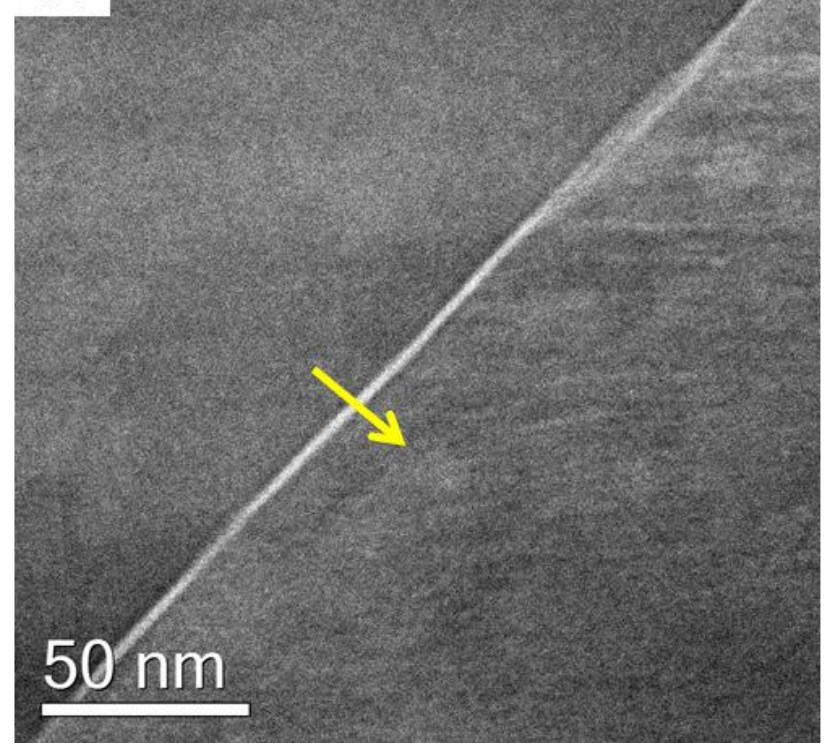

Figure 3 An EDS composition line scan in Cs-corrected HAADF STEM (a) shows an increase in Mn concentration (at.\%) at a grain boundary, which is imaged in (b). The start and finish direction of the scan is marked with the yellow arrow in (b). The average concentration of the line scan is 6.9 at.\%, and the concentration at the boundary is about 10.5 at.\% on average. 
(a)

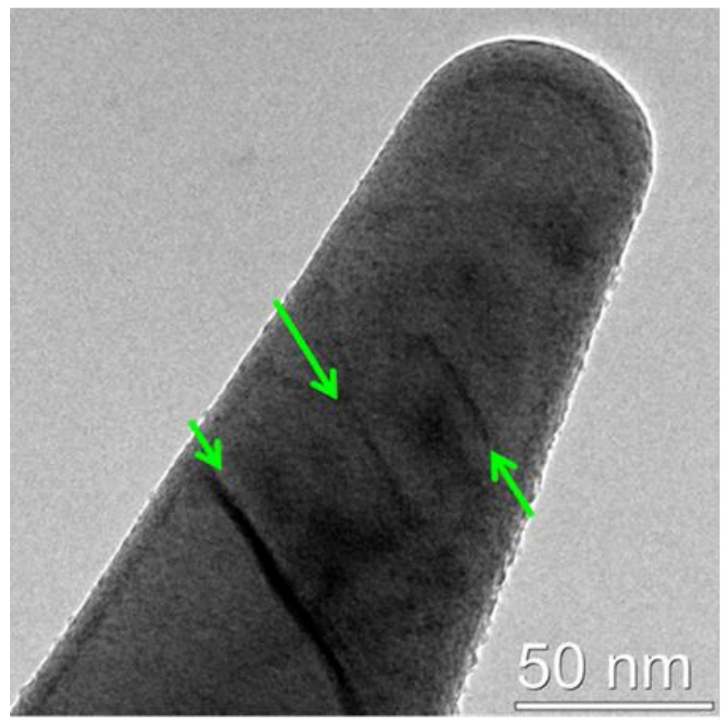

(b)

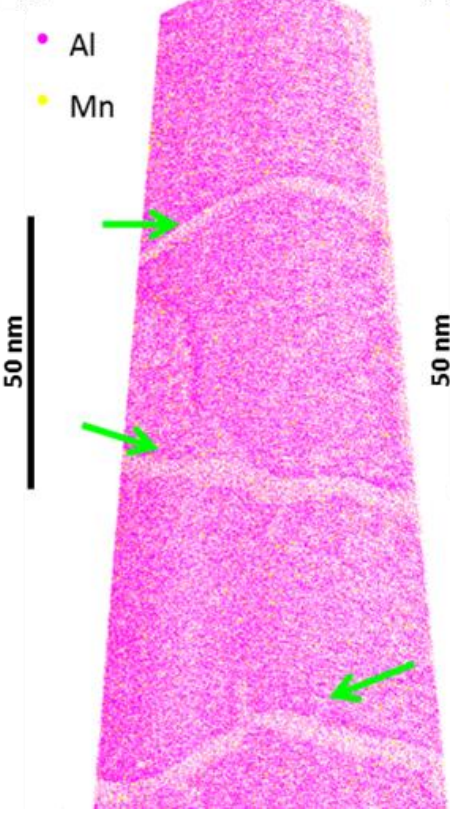

(c)

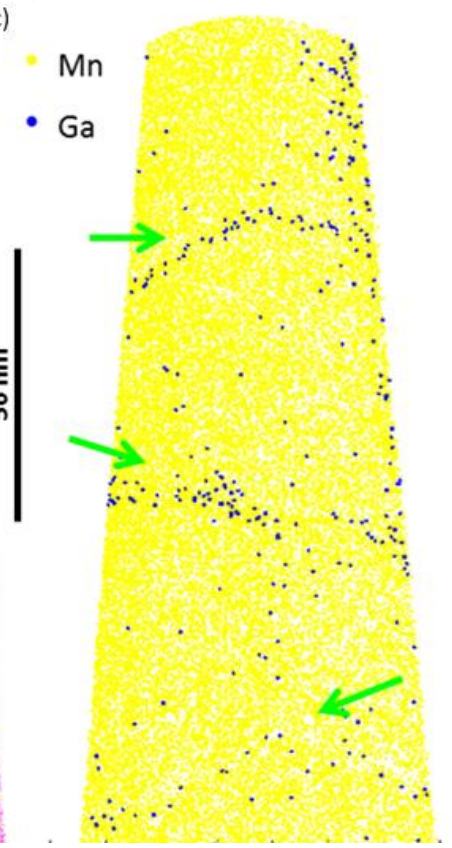

Figure 4 (a) TEM picture of an APT sample. Green arrows mark the position of three individual grain boundaries. (b) The atom dot maps of $\mathrm{Al}$ and $\mathrm{Mn}$ and (c) $\mathrm{Mn}$ and $\mathrm{Ga}$ for the same sample. 

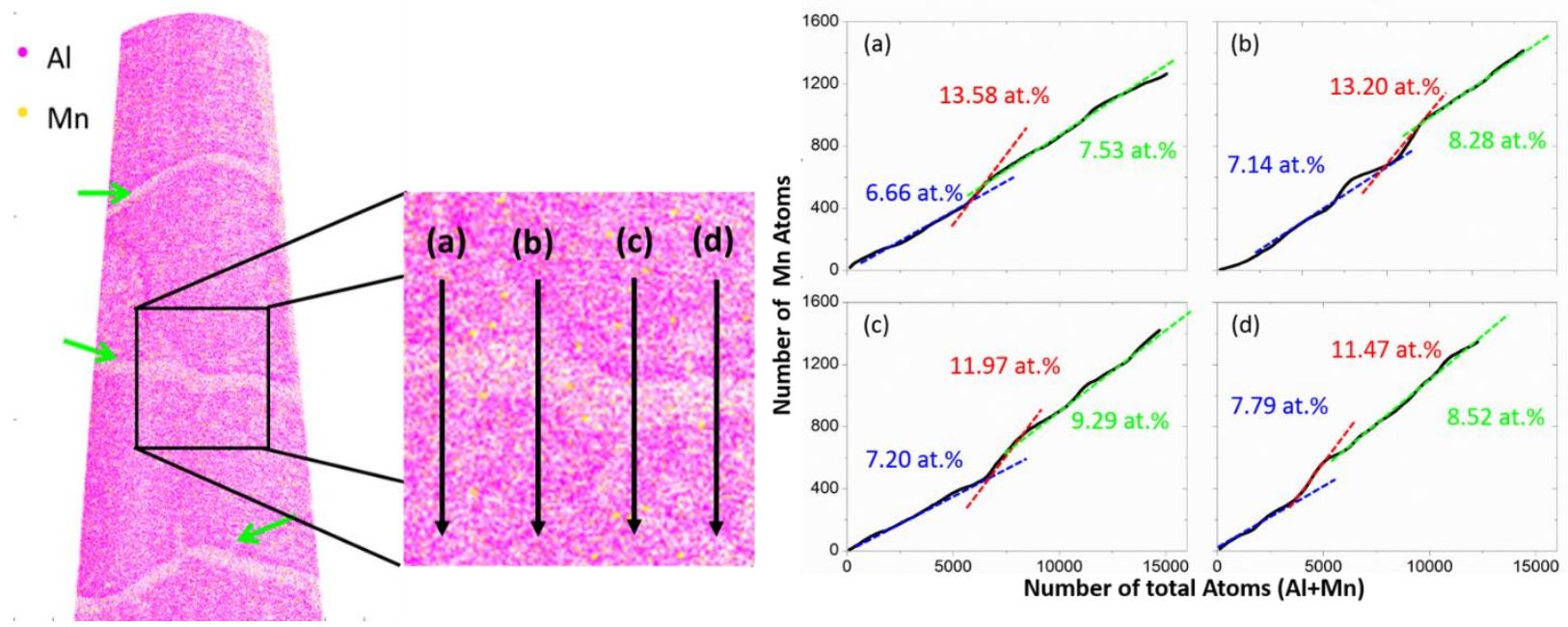

Figure 5 Ladder diagram analysis of an individual grain boundary on the atom probe sample.

Each line here represents a one dimensional $30 \mathrm{~nm}$ scan across the marked grain boundary, with an $1.8 \times 1.8 \mathrm{~nm}^{2}$ cross sectional area. The $\mathrm{x}$ axis is the cumulative total $(\mathrm{Al}+\mathrm{Mn})$ number of atoms while the $\mathrm{y}$ axis is the accumulated number of $\mathrm{Mn}$ atoms encountered in a one-dimensional traverse across the boundary. The slopes marked here represent the Mn concentration for each scan. The slopes all change across the grain boundary. (The blue dashed line and green dashed line show the concentrations for grain interiors, and the red dashed line denotes the region of higher slope that corresponds to the grain boundary.) The grain boundary is enriched by about 3.6 at.\% Mn in line (d) to 6.92 at.\% Mn in line (a) as compared with the background level in the grains. 

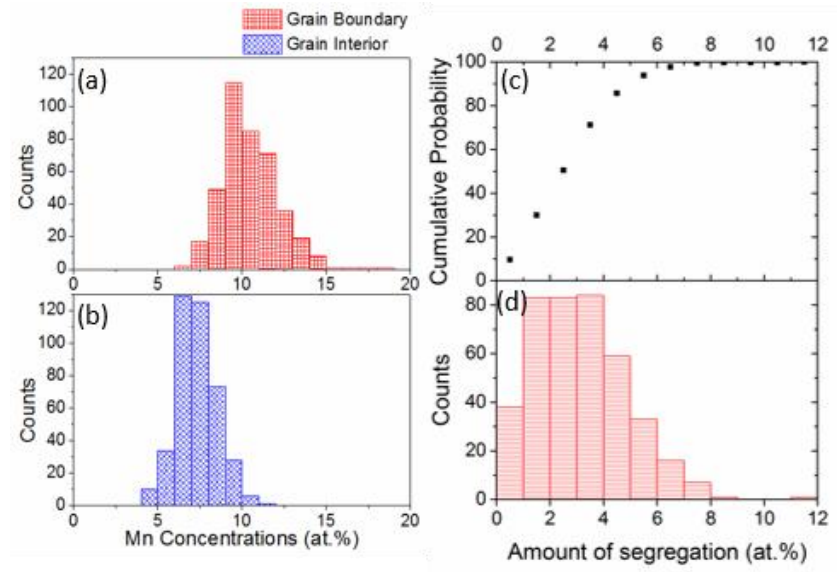

Figure 6 Concentration distributions for regions in the (a) grain boundary and (b) grain interior of an APT sample. The average grain boundary concentration is 10.5 at.\% $\mathrm{Mn}$ and the average concentration of the grain interior is 7.3 at.\%. The degree of segregation (concentration difference between grain boundary and grain interior) is also plotted with a cumulative probability plot in (c) and histogram in (d). The average degree of segregation is 3.1 at.\% Mn for this grain boundary. 
(a)

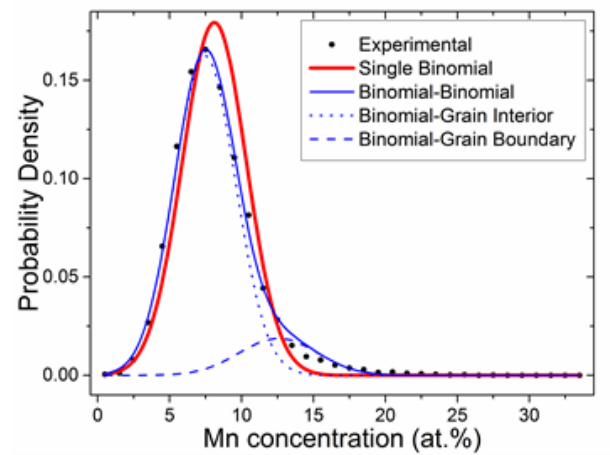

(b)

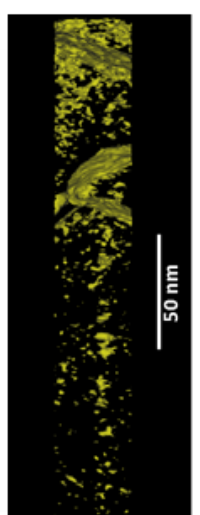

Figure 7 (a) Frequency distribution for one of the atom probe samples with a volume of $36 \times 16$ $\times 234 \mathrm{~nm}^{3}$, divided up into blocks containing $\mathrm{n}=200$ atoms each, and compared with single binomial fitting and binomial-binomial fitting. The black dots show the experimental data frequency distribution. The single binomial fitting (red) significantly deviates from the experimental data, which is broader; suggesting the distribution of solute is not random. The data is further fitted with a dual-binomial function (solid blue line), with sub-distributions shown with dashed lines (b) The corresponding iso-concentration surface at $\mathrm{c}=10.5 \% \mathrm{Mn}$ for the volume analyzed in (a). 
Table 1 Results of effective heat of segregation $\left(\mathrm{H}_{\mathrm{seg}}\right)$ extracted from experimental data at room temperature using McLean isotherm analysis.

\begin{tabular}{|l|c|}
\hline Methods & $\mathrm{H}_{\mathrm{seg}}\left(\mathrm{J} \mathrm{mol}^{-1}\right)$ \\
\hline STEM (line scan measurement) & 1145 \\
\hline STEM (area density measurement)* & 1177 \\
\hline APT (line scan average) & 1262 \\
\hline APT (binomial fitting) & 1420 \\
\hline Miedema based model (with $\left.\Delta H_{\mathrm{BinA}}^{\text {int }}\right)$ & 1030 \\
\hline Miedema based model (with $\left.\Delta H_{\mathrm{RKM}}^{\text {int }}\right)$ & 5280 \\
\hline
\end{tabular}

*assumes grain boundary thickness in STEM $=1 \mathrm{~nm}$. 
Table 2 Numerical values for calculation of grain boundary energy from Miedema [60], de Boer [61] and James et al. [62]

\begin{tabular}{|l|l|l|l|}
\hline Parameter & Value & Parameter & Value \\
\hline$\Delta H_{\mathrm{BinA}}^{\text {int }}$, Miedema $\left(\mathrm{k} \mathrm{J} \mathrm{mol}^{-1}\right)$ & 106 & $V_{A}(\mathrm{~A}=\mathrm{Al})\left(10^{-6} \mathrm{~m}^{3} \mathrm{~mol}^{-1}\right)$ & 10.00 \\
\hline$\Delta H_{\mathrm{RKM}}^{\text {int }}, \mathrm{Calphad}\left(\mathrm{k} \mathrm{J} \mathrm{mol}^{-1}\right)$ & 70 & $\left.V_{B}(\mathrm{~B}=\mathrm{Mn})\right)\left(10^{-6} \mathrm{~m}^{3} \mathrm{~mol}^{-1}\right)$ & 7.35 \\
\hline$\gamma_{\mathrm{A}}^{\mathrm{S}},(\mathrm{A}=\mathrm{Al}),\left(\mathrm{J} \mathrm{m}^{-2}\right)$ & 1.16 & $K_{\mathrm{A}}(\mathrm{A}=\mathrm{Al})(\mathrm{GPa})$ & 75.50 \\
\hline$\gamma_{\mathrm{B}}^{\mathrm{S}},(\mathrm{B}=\mathrm{Mn}),\left(\mathrm{J} \mathrm{m}^{-2}\right)$ & 1.60 & $G_{\mathrm{B}}(\mathrm{B}=\mathrm{Mn})(\mathrm{GPa})$ & 76.50 \\
\hline
\end{tabular}

